\title{
Synthesis and electromagnetic properties of FeNi alloy nanofibers using an electrospinning method
}

\author{
Young-In Lee* and Yong-Ho Choa***,† \\ *Institute of Nano Sensor Technology, Hanyang University, Ansan 426-791, Korea \\ **Department of Fusion Chemical Engineering, Hanyang University, Ansan 426-791, Korea \\ (Received September 20, 2012) \\ (Revised October 8, 2012) \\ (Accepted October 12, 2012)
}

\begin{abstract}
FeNi}$ alloy nanofibers have been prepared by an electrospinning process followed by air-calcination and $\mathrm{H}_{2}$ reduction to develop electromagnetic (EM) wave absorbers in the giga-hertz $(\mathrm{GHz})$ frequency range. The thermal behavior and phase and morphology evolution in the synthetic processes were systematically investigated. Through the heat treatments of calcination and $\mathrm{H}_{2}$ reduction, as-spun PVP/FeNi precursor nanofiber has been stepwise transformed into nickel iron oxide and FeNi phases but the fibrous shape was maintained perfectly. The FeNi alloy nanofiber had the high aspect ratio and the average diameter of approximately $190 \mathrm{~nm}$ and primarily composed of FeNi nanocrystals with an average diameter of $\sim 60 \mathrm{~nm}$. The FeNi alloy nanofibers could be used for excellent EM wave absorbing materials in the $\mathrm{GHz}$ frequency range because the power loss of the FeNi nanofibers increased up to $20 \mathrm{GHz}$ without a degradation and exhibited the superior EM wave absorption properties compared to commercial FeNi nanoparticles.
\end{abstract}

Key words Nanofiber, Electrospinning, FeNi alloy, EM absorber, Electromagnetic Properties

\section{Introduction}

Electromagnetic (EM) waves with the $\mathrm{GHz}$ band are widely used for electronic devices such as wireless telecommunication systems and high-frequency circuit devices in commercial, industrial, and military applications, and the frequency used for these devices will shift to the high-frequency range of above $10 \mathrm{GHz}$ in the future [14]. Therefore, EM wave absorbers suitable for these frequency bands are strongly required for the suppressing of EM interference between the component and signal source. Studies have focused on effective electromagnetic (EM) wave absorbing materials that have a wide absorption frequency, high absorption capability, low weight, good thermal stability, and anti-oxidation capability.

In general, the shielding efficiency of EM wave absorbers depends on the matching frequency, layer thickness of absorbers, and the relative complex permeability and permittivity of the fillers which are determined by their nature, shape, size, and microstructure [5-7]. Metallic soft magnetic materials may be more suitable as the EM wave absorbers in the frequency range over $\mathrm{GHz}$ com-

\footnotetext{
Corresponding author

Tel: +82-31-400-5650

Fax: +82-31-418-6490

E-mail: choa15@hanyang.ac.kr
}

pared to the conventional ferrites because they possess a higher saturation magnetization and permeability that can still have a large value in a wide $\mathrm{GHz}$ range which results from their higher Snoek's limits [6-8].

Among the metallic soft magnetic materials, FeNi alloys show excellent soft magnetic properties, and have been widely applied in the field of electronic devices and industry $[9,10]$. In addition, nano-sized ferromagnetic materials with high aspect ratio (i.e., one-dimensional (1D) nanostructure) including nanorods, nanowires, and nanofibers can lead to high permittivity and permeability at $\mathrm{GHz}$ frequency ranges [11, 12]. Among various synthetic techniques for 1D nanostructures, electrospinning is a simple and versatile method that can be used to easily fabricate various $1 \mathrm{D}$ nanostructures with controllable diameters, compositions and morphologies on an industrial scale [13-16].

In this study, FeNi alloy nanostructures with a high aspect ratio were prepared by electrospinning method followed by air-calcination and $\mathrm{H}_{2}$ reduction. The thermal behavior and phase and morphology evolution in the synthetic processes were systematically investigated. The EM characteristics of the 1D FeNi nanostructure and commercial FeNi nanoparticles were evaluated and compared to determine the usefulness of the 1D FeNi nanostructure as an EM wave absorbing material in $\mathrm{GHz}$ 
frequency range.

\section{Experimental Details}

First, $2.908 \mathrm{~g}$ of nickel (II) nitrate hexahydrate $\left(\mathrm{Ni}\left(\mathrm{NO}_{3}\right)_{2}\right.$. $6 \mathrm{H}_{2} \mathrm{O}, 99.9 \%$, High Purity Chemicals) and $4.040 \mathrm{~g}$ of iron (III) nitrate nonahydrate $\left(\mathrm{Fe}\left(\mathrm{NO}_{3}\right)_{3} \cdot 9 \mathrm{H}_{2} \mathrm{O}, 99.9 \%\right.$, High Purity Chemicals) with Ni/Fe molar ratio of $1: 1$ together with $0.5 \mathrm{~g}$ polyvinyl pyrrolidone (PVP, $\mathrm{Mw}=$ $1,300,000 \mathrm{~g} / \mathrm{mol}$, Aldrich) were dissolved in a mixture of $3 \mathrm{~m} l$ of distilled water and $12 \mathrm{~m} l$ of ethanol under magnetic stirring to form a homogeneous viscous solution. The solution was loaded into a plastic syringe with a nozzle connector with a capillary tip $(0.14 \mathrm{~mm}$ diameter) at the end and fed at a constant rate of $0.3 \mathrm{ml} / \mathrm{hr}$. A voltage of $16 \mathrm{kV}$ was applied between the capillary tip and the collector using flat aluminum foil at a distance of $10 \mathrm{~cm}$. The electrospun PVP/FeNi precursor nanofibers were prepared by electrospinning and then the nickel iron oxide nanofiber was obtained by annealing the as-spun nanofibers at $600^{\circ} \mathrm{C}$ for $2 \mathrm{~h}$ in air with a heating rate of $5^{\circ} \mathrm{C} / \mathrm{min}$. The nickel iron oxide nanofibers were reduced to $\mathrm{FeNi}$ nanofiber by thermal treatment at $500^{\circ} \mathrm{C}$ for $1 \mathrm{~h} \mathrm{in} \mathrm{H}_{2}$, using a heating rate of $5^{\circ} \mathrm{C} /$ min.

The thermal behaviors of the PVP/FeNi precursor nanofiber and the nickel iron oxide nanofiber were investigated by thermogravimetric analysis (TGA, SDTQ-600, TA Instruments Co.). The morphological investigation and structural analysis of the nanofibers were performed using a field emission scanning electron microscope (FE-SEM, Hitachi S-4800) and a transmission electron microscope (TEM, JEOL JEM-2100F). X-ray diffractometer (XRD, Rigaku, D/MAX-2500/PC) and selected area diffraction pattern (SAED) were used to determine the crystal structure of the synthesized nanofibers. Finally, the epoxy matrix composites containing the $30 \mathrm{wt} \%$ FeNi nanofibers and FeNi nanoparticles were fabricated by shear mixing method. The composites were compacted into a square sheet $(4 \mathrm{~mm} \times 4 \mathrm{~mm} \times 200 \mu \mathrm{m})$ by an auto-press (Carver, USA). Electromagnetic wave absorption abilities of the electrospun FeNi nanofiber and the FeNi nanoparticles were calculated by S-parameters (S11, S21), which were measured by a network analyzer (Vector Network Analyzer System, Anritsu 37247D) with a coplanar waveguide (CPW) that is designed with a $100 \mu \mathrm{m}$-wide signal line and $50 \Omega$ characteristic impedance.

\section{Results and Discussion}

Fig. 1(a) shows a TG curve of the as-spun PVP/FeNi precursor nanofibers. The weight loss between $50^{\circ} \mathrm{C}$ and $270^{\circ} \mathrm{C}$ is considered to result from the removal of solvent and PVP side chain. It was also thought that the Fe and Ni nitrate and PVP main chain were decomposed between $300^{\circ} \mathrm{C}$ and $550^{\circ} \mathrm{C}$ and this was corresponded to the peak of DTG. The weight loss of the precursor fiber was $66 \%$ to remain about $20 \%$ of nickel iron oxide. The curve indicated that all of the organics and volatiles, with the exception of $\mathrm{Fe}-\mathrm{Ni}$ based products, were decomposed and removed below $550^{\circ} \mathrm{C}$. Therefore, based on the TG analysis results, the thermal treatment temperatures for the removal of organics and volatiles were determined to $600^{\circ} \mathrm{C}$. Fig. 1(b) shows a TG curve of the $\mathrm{Fe}-\mathrm{Ni}$ based products in $\mathrm{H}_{2}$ atmosphere. The weight loss started at $340^{\circ} \mathrm{C}$ and finished around $400^{\circ} \mathrm{C}$. This was attributed to the reduction of nickel iron oxide to FeNi alloy. The weight loss was about $26 \%$ which is consistent with the loss of 2.5 mole of oxygen. There-
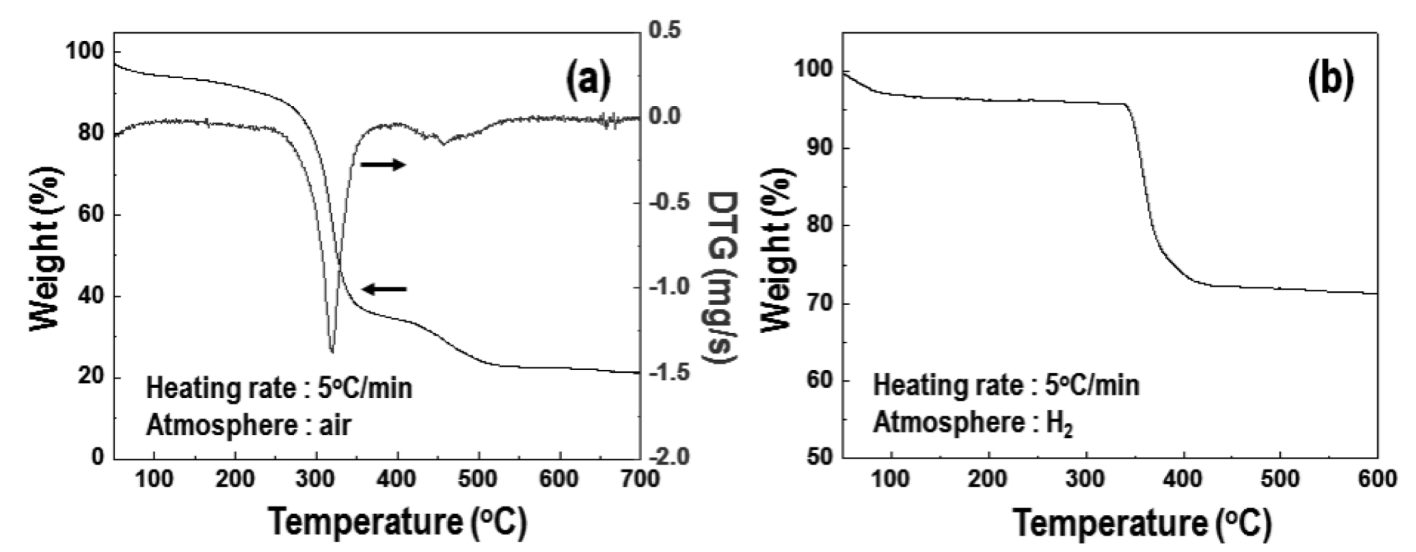

Fig. 1. Thermo-gravimetric analysis (TGA) curve of (a) PVP/FeNi precursor nanofibers in air and (b) Fe-Ni oxide nanofibers in $\mathrm{H}_{2}$. 


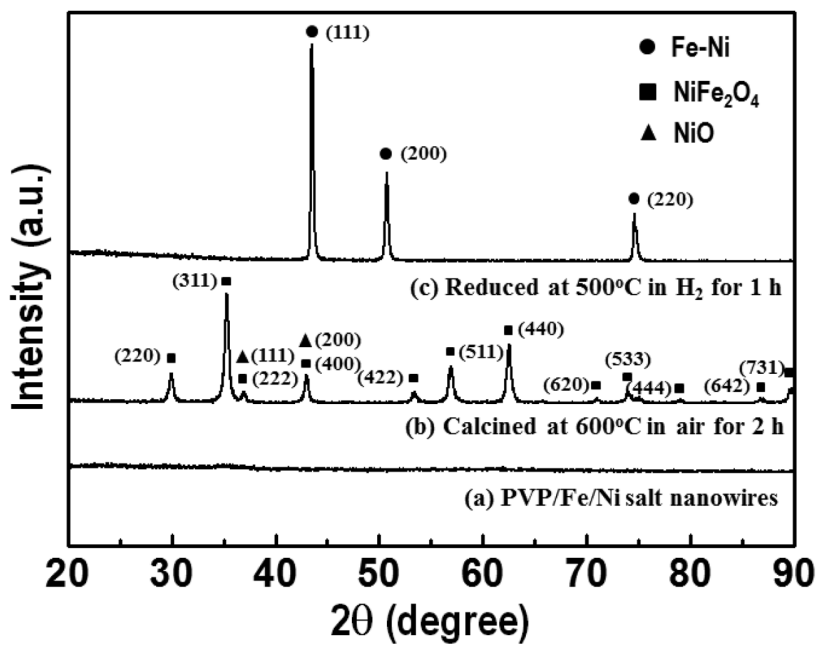

Fig. 2. XRD patterns for the (a) as-prepared, (b) air-calcined, and $(c)$ reduced nanofibers.

fore, the reduction to the FeNi alloy were determined to $500^{\circ} \mathrm{C}$.

XRD patterns for the as-prepared, air-calcined, and reduced nanofibers are shown in Fig. 2. The as-prepared $\mathrm{PVP} / \mathrm{FeNi}$ precursor nanofiber was amorphous but the nanofiber calcined at $600^{\circ} \mathrm{C}$ for $2 \mathrm{~h}$ in air showed wellcrystallized cubic $\mathrm{NiFe}_{2} \mathrm{O}_{4}$ with a spinel structure (JCPDS card No. 10-0325) and NiO(JCPDS card No. 44-1159). As shown in Fig. 2(c), the nickel iron oxide was completely reduced to face-centered cubic FeNi alloy (JCPDS card No. 47-1417) by thermal treatment at $500^{\circ} \mathrm{C}$ for $1 \mathrm{~h}$ in $\mathrm{H}_{2}$.

The effect of the calcination and reduction on the morphology and diameter of the nanofibers was investi- gated by FE-SEM. Fig. 3(a c) shows FE-SEM images of the as-prepared, air-calcined, and reduced nanofibers with diameter distribution graphs in Fig. 3(d f). As shown in Fig. 3(a), the nanofibers had a high aspect ratio and a smooth surface without any beads. The average diameter of the nanofibers was measured to be $340 \mathrm{~nm}$ based on the FE-SEM images. After thermal treatment in air at $600^{\circ} \mathrm{C}$ for $2 \mathrm{~h}$, the fibrous shape was maintained perfectly and the surface was much rougher than the as-prepared nanofibers due to the crystallization of nickel iron oxide. The average diameter of the nickel iron oxide nanofibers decreased to approximately $218 \mathrm{~nm}$ due to the elimination of PVP. The volume contraction of as-spun fibers to nickel iron oxide fibers was $59 \%$, based on the calculation of decrease of average diameter. The most important one is that the fibrous shape was also maintained after thermal treatment in $\mathrm{H}_{2}$ despite their high volume contraction to result in the successful synthesis of FeNi alloy nanofibers (Fig. 3(c)). The average diameter of the FeNi nanofibers is slightly decreased compared to that of the nickel iron oxide nanofibers (Fig. 3(f)). The difference in diameter is the result of shrinkage during the reduction from nickel iron oxide nanofibers to FeNi nanofibers.

Fig. 4(a) displays a TEM image for the FeNi nanofiber. The nanofibers had a rough surface throughout their entire length and a high aspect ratio. In addition, the nanofibers primarily composed of FeNi nanocrystals with an average diameter of $\sim 60 \mathrm{~nm}$. The SAED pattern of the nanofibers demonstrated that they are polycrystalline with the d-spacing consistent with XRD results. In
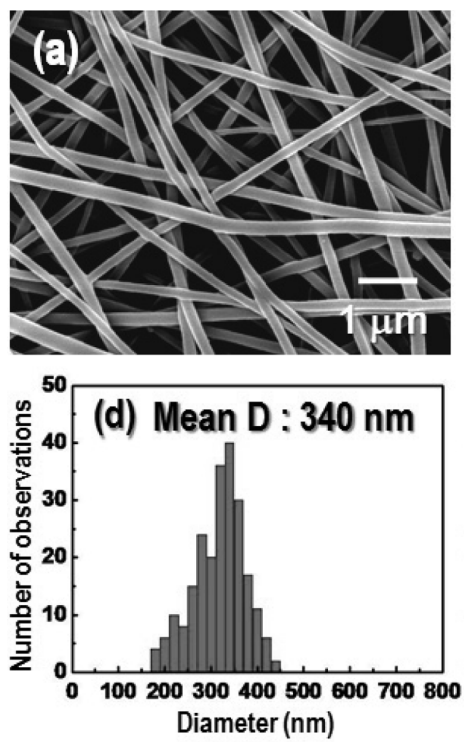
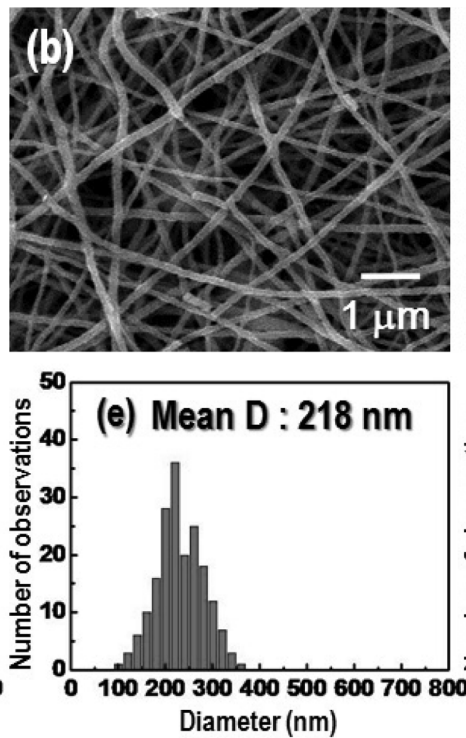
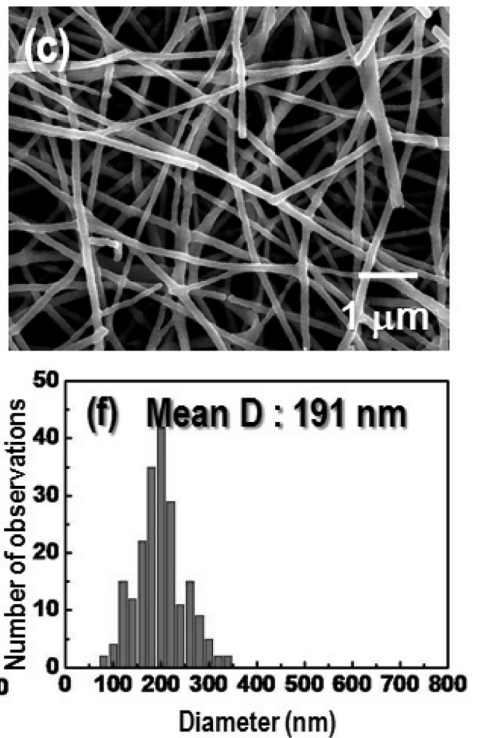

Fig. 3. FE-SEM images and diameter distribution graphs of the nanofibers; (a), (d) as prepared PVP/FeNi precursor nanofibers, (b), (e) nickel iron oxide nanofibers and (c), (f) FeNi nanofibers. 

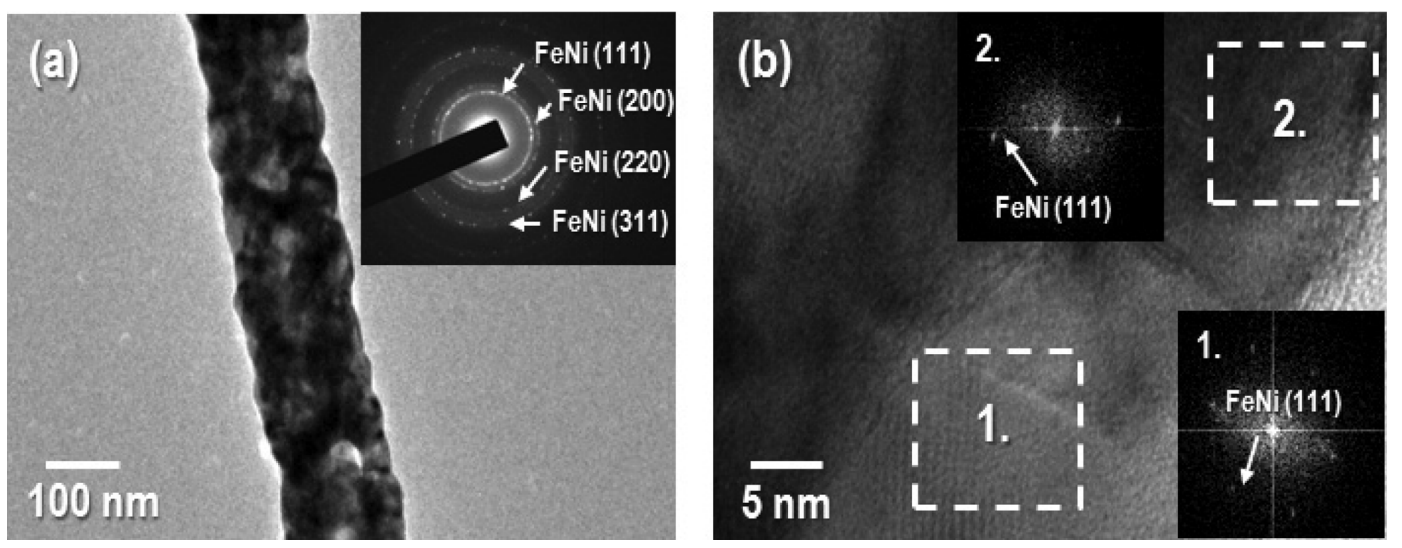

Fig. 4. (a) TEM images with SAED pattern and (b) HR-TEM with FFT analysis of the FeNi nanofiber.

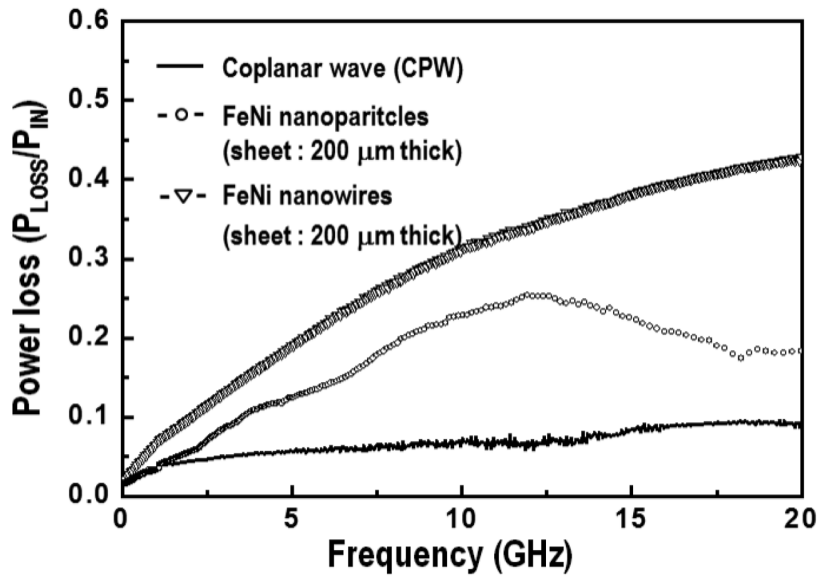

Fig. 5. Graphs showing the power loss of FeNi nanofibers and nanoparticles calculated from S-parameters.

Fig. 4(b), fast Fourier transform (FFT) analysis was performed on the lattice fringe and the diffraction spots well matched the (111) lattice plane of FeNi alloy.

Fig. 5 shows the power loss of a $200-\mu$ m-thick composite sheet filled with FeNi nanofibers and commercial FeNi nanoparticles (> $100 \mathrm{~nm}$, Sigma-Aldrich). The power loss can be calculated by the measured S-parameter using following equation, $\mathrm{P}_{\mathrm{LOSS}} / \mathrm{P}_{\mathrm{IN}}=1-\left(\left|\mathrm{S}_{11}\right|^{2}+\left|\mathrm{S}_{21}\right|^{2}\right)$, where $S_{11}$ and $S_{21}$ are the reflection and transmission coefficients, respectively. The power loss of the commercial sheets increased incrementally as the frequency increased up to around $10 \mathrm{GHz}$. On the other hand, the power loss of the FeNi nanofiber based composite sheet increased as the frequency increased up to $20 \mathrm{GHz}$. These results indicate that high aspect ratio FeNi nanofibers improve the electromagnetic wave absorption compared to low aspect ratio FeNi nanoparticles and can be explained that in case of using the high aspect ratio of the fillers, the formations of bigger electric and magnetic dipoles and conductive networks became more feasible [25]. This finding implies that the FeNi nanofiber can be used as the efficient EM absorber material in the $\mathrm{GHz}$ frequency range.

\section{Conclusion}

In conclusion, in order to increase the EM wave absorbing properties in the $\mathrm{GHz}$ frequency range, the FeNi nanofibers with high aspect ratio have been successfully prepared by the multi-nozzle electrospinning process followed by air-calcination and $\mathrm{H}_{2}$ reduction. The FeNi alloy nanofiber had the high aspect ratio and the average diameter of approximately $190 \mathrm{~nm}$ and primarily composed of FeNi nanocrystals with an average diameter of $\sim 60 \mathrm{~nm}$. The FeNi alloy nanofibers could be used for excellent EM wave absorbing materials in the $\mathrm{GHz}$ frequency range because the power loss of the FeNi nanofibers increased up to $20 \mathrm{GHz}$ without a degradation and exhibited the superior EM wave absorption properties compared to FeNi nanoparticles.

\section{Acknowledgment}

This research was supported by Basic Science Research Program through the National Research Foundation of Korea (NRF) (2008-0061861) and the Pioneer Research Center Program through the National Research Foundation of Korea (2011-0001645) funded by the Ministry of Education, Science and Technology (MEST).

\section{References}

[1] S. Kimura, T. Kato, T. Hyodo, Y. Shimizu and M. 
Egashira, "Electromagnetic wave absorption properties of carbonyl iron-ferrite/PMMA composites fabricated by hybridization method", J. Magn. Magn. Mater. 312 (2007) 181.

[2] J. Wei, J. Liu and S. Li, "Electromagnetic and microwave absorption properties of $\mathrm{Fe}_{3} \mathrm{O}_{4}$ magnetic films plated on hollow glass spheres", J. Magn. Magn. Mater. 312 (2007) 414.

[ 3 ] S.W. Phang, M. Tadokoro, J. Watanabe and N. Kuramoto, "Microwave absorption behaviors of polyaniline nanocomposites containing $\mathrm{TiO}_{2}$ nanoparticles", Curr. Appl. Phys. 8 (2008) 391.

[4] S. Sugimoto, T. Maeda, D. Book, T. Kagotani, K. Inomata, M. Homma, H. Ota, Y. Houjou and R. Sato, " $\mathrm{GHz}$ microwave absorption of a fine $\alpha-\mathrm{Fe}$ structure produced by the disproportionation of $\mathrm{Sm}_{2} \mathrm{Fe}_{17}$ in hydrogen", J. Alloys Compd. 330 (2002) 301.

[ 5 ] Y. Deng, L. Zhao, B. Shen, L. Liu and W. Hu, "Microwave characterization of submicrometer-sized nickel hollow sphere composites", J. Appl. Phys. 100 (2006) 14304.

[6 ] J.R. Liu, M. Itoh and K.I. Machida, "Magnetic and electromagnetic wave absorption properties of $\alpha$-Fe/Z-type Ba-ferrite nanocomposites", Appl. Phys. Lett. 88 (2006) 062503.

[ 7 ] X.F. Zhang, X.L. Dong, H. Huang, Y.Y. Liu, W.N. Wang, X.G. Zhu, B. Lv, J.P. Lei and C.G. Lee, "Microwave absorption properties of the carbon-coated nickel nanocapsules", Appl. Phys. Lett. 89 (2006) 053115.

[ 8 ] W.F. Yang, L. Qiao, J.Q. wei, Z.Q. Zhang, T. Wang and F.S. Li, "Microwave permeability of flake-shaped $\mathrm{FeCuNb}$ $\mathrm{SiB}$ particle composite with rotational orientation", J. Appl. Phys. 107 (2010) 033913.
[9] I. Chicinas, O. Geoffroy, O. Isnard and V. Pop, "AC magnetic properties of the soft magnetic composites based on nanocrystalline $\mathrm{Ni}-\mathrm{Fe}$ powders obtained by mechanical alloying”, J. Magn. Magn. Mater. 310 (2007) 2474

[10] Y. Shirakata, N. Hidaka, M. Ishitsuka, A. Teramoto and T. Ohmi, "High permeability and low loss Ni-Fe composite material for high-frequency applications", IEEE Trans. Magn. 44 (2008) 2100.

[11] M.S. Kim, E.H. Min and J.G. Koh, "Comparison of the effects of particle shape on thin $\mathrm{FeSiCr}$ electromagnetic wave absorber", J. Mag. Mag. Mater. 321 (2009) 581.

[12] M. Itoh, J.R. Liu, T. Horikawa and K. Machida, "Electromagnetic wave absorption properties of nanocomposite powders derived from intermetallic compounds and amorphous carbon", J. Alloy Comp. 408 (2006) 1400.

[13] D. Li and Y. Xia, "Fabrication of titania nanofibers by electrospinning", Nano Lett. 3 (2003) 555.

[14] C.-G. Song and J.-W. Yoon, "Optical properties of Al doped $\mathrm{ZnO}$ nanofibers prepared by electrospinning", J. Kor. Cryst. Growth and Cryst. Technol. 21 (2011) 205.

[15] J.Y. Hwang, H.J. Kim, N.-H. Park, H. Huh, C.K. Park and J.-W. Yoon, "Fabrication of $\mathrm{PLA} / \mathrm{TiO}_{2}$ nanofibers using melt-electro-spinning", J. Kor. Cryst. Growth and Cryst. Technol. 21 (2011) 124.

[16] S. Cavaliere, S. Subianto, L. Chevallier, D.J. Jones and J. Roziere, "Single step elaboration of size-tuned Pt loaded titania nanofibres", Chem. Commun. 47 (2011) 6834.

[17] S.G. Shin and S.R. Lee, "Effect of aspect ratio on percolation structure of two-phase composites with conductivity”, Korean J. Met. Mater. 48 (2010) 581. 